Editor's Note: These short, critical reviews of recent papers in the Journal, written exclusively by graduate students or postdoctoral fellows, are intended to summarize the important findings of the paper and provide additional insight and commentary. For more information on the format and purpose of the Journal Club, please see http://www.jneurosci.org/misc/ifa_features.shtml.

\title{
Practice Makes Cortex
}

\author{
Emma G. Duerden and Danièle Laverdure-Dupont \\ Département de physiologie, Université de Montréal, Montréal, Québec, Canada H3C 3J7, and Centre de recherche de l’institut universitaire de gériatrie de \\ Montréal, Montréal, Québec, Canada H3W 1W4 \\ Review of Ilg et al. (http://www.jneurosci.org/cgi/content/full/28/16/4210)
}

With the development of high-resolution anatomical magnetic resonance imaging (MRI) and automated statistical analysis techniques, brain morphometry has emerged as a dynamic field in neuroscience. A variety of techniques are now used to assess gray and white matter characteristics, such as thickness and density. Additionally, intrinsic changes in cortical folding can be explored using gyrification and sulcal morphometric analyses. To date, morphometric research has contributed significantly to our understanding of neurodegenerative diseases as well as experience-dependent brain plasticity.

Traditionally, it was believed that the organization of the cortex was static. However, results from the elegant work of Merzenich and colleagues (for review, see Merzenich and Sameshima, 1993) in nonhuman primates has demonstrated the presence of experience-dependent plasticity in the parietal cortex in response to somatosensory training. This extensive remapping was paralleled with improvements in behavioral performance. Findings from animal models have widened neuroscientists' views of the dynamic nature of the brain and have underscored the importance of verifying the presence and functional implications of such processes in humans.

Received July 17, 2008; revised July 11, 2008; accepted July 11, 2008.

Correspondence should be addressed to Emma G. Duerden, Département de physiologie, Groupe de recherche sur le système nerveux central, Université de Montréal, C.P. 6128, Succ A, Montréal, Québec, Canada H3C 3J7.E-mail: emma.duerden@umontreal.ca.

DOI:10.1523/JNEUROSCI.2650-08.2008

Copyright $\odot 2008$ Society for Neuroscience $\quad$ 0270-6474/08/288655-03\$15.00/0
Voxel-based morphometry is a modern, noninvasive computational approach allowing the study of neuroanatomical changes in living human subjects. This technique involves voxelwise measures of local concentrations of gray or white matter (Ashburner and Friston, 2000). In initial steps, MRI data are registered to a standardized template, tissues types are segmented with regards to their intensities, and the resulting images are smoothed. Parametric statistical tests are then used to examine divergences between different cohorts of subjects or longitudinal changes within a single population. Another major advantage of voxel-based morphometry is the possibility of correlating changes in anatomical structure with the modulation of cerebral activation, as measured with a variety of functional imaging techniques.

Several morphometric studies have demonstrated both short- and long-term use-dependent changes in the cortex. Voxel-based morphometry has been used to study experience-related plasticity in response to explicit tasks such as spatial navigation (Maguire et al., 2000) or intensive studying (Draganski et al., 2006) and implicit tasks including language skill learning (Mechelli et al., 2005), musical training (Gaser and Schlaug, 2003), and juggling (Draganski et al., 2004). Although structural changes are commonly found in brain regions known to be functionally involved in the particular skill under study, a meta-analytic review of these studies revealed that additional changes often occurred in associative regions in- cluding parietal and temporal cortices (Fig. 1).

The link between functional and anatomical cortical plasticity has remained essentially unexplored. With the advent of computational analysis, it is now possible to spatially correlate loci of functional activation with emerging morphological modifications induced by practice. Ilg et al. (2008) examined this question by combining functional MRI (fMRI) and anatomical MRI to study practice-induced cortical plasticity in response to a procedural learning task. Participants assigned to the experimental group practiced a mirror reading task for 15 min every day over a 2 week period. Subjects' behavioral performance at reading mirrored words was recorded before and after 1 week and again after 2 weeks of training. Practicerelated performance increases in mirrorreading were observed as soon as after 1 week of practice, with slight additional improvement in the following week [Ilg et al. (2008), their Fig. 1 (http://www. jneurosci.org/cgi/content/full/28/16/ 4210/F1)]. Functional and structural imaging was performed only before the initiation of training and after 2 weeks of practice. To examine functional changes, brain activation was compared during the reading of mirrored words before and after training. Significant training-related increases were observed in the blood oxygen level-dependent signal in the right dorsal occipital cortex and left thalamus. Additionally, a significant decrease was noted in the right superior parietal cortex. In turn, voxel-based morphometry analy- 
sis was used to explore structural gray matter density changes that occurred before and after training. Although no decreases were detected between training days, a significant increase in gray matter density was detected in the right dorsal occipital cortex, localized to the peak activation increase observed in the fMRI data. In line with previous findings, these results suggest that, after practice, structural changes occurred in areas that were actively recruited during practice at the task. Because behavioral data showed a steep increase after only 1 week of training, a direct comparison of anatomical and functional images collected in the same temporal window might have better clarified the relationship between such changes. Although practice-related changes in fMRI activation and gray matter density were localized in visual areas, the change in the fMRI activation was reported in the right $\mathrm{V} 1$ [primary occipital cortex; Brodmann's area (BA)17/18], whereas the peak region of increased gray matter density after training was slightly more lateral and encroached on higherorder visual processing areas (BA18/19) [Ilg et al. (2008), their Fig. 2 (http:// www.jneurosci.org/cgi/content/full/28/ $16 / 4210 / F 2)]$. In light of the reduced time required to accomplish mirrored reading, one can postulate that, in the early stage of practice, complex visual processing involving mental translation of the mirrored letters was needed to enable comprehensive detection. As the time required to accomplish the task diminished, subjects might have gradually relied on memorized mirrored letters without the need for abstract transformation. Hence, activation of primary visual areas noted after 2 weeks of practice could reflect a stage at which a more automated process based on simple mirrored letter recognition was used. Under those assumptions, whereas early phases of learning could have relied mainly on associative visual areas, later stages of automated detection might have only required the activation of primary visual regions. In this context, the increase in gray matter density of higher-order visual areas could reflect the changes that occurred earlier in the learning process, when the task was still cognitively demanding. However, strategies may have differed among participants, hence the need for correlating functional and structural changes within individuals.

In a voxel-based morphometric analysis, the tissue composition of each voxel is inferred from differences in image inten-
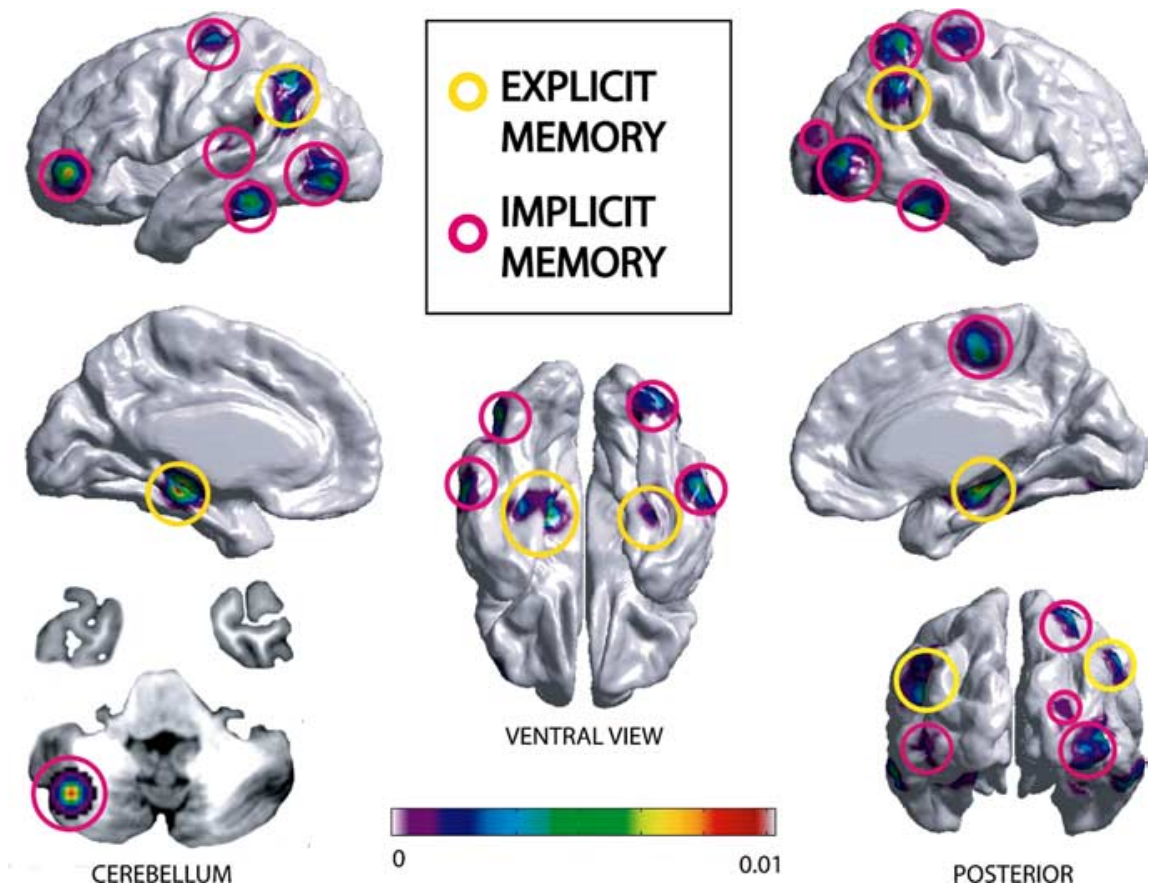

Figure 1. Meta-analysis of voxel-based morphometric studies reporting increased gray matter density after learning in the cortex and cerebellum. Factual knowledge that is recalled by a purposeful effort requires the involvement of the explicit memory system. It is involved in tasks such as spatial navigation (Maguire et al., 2000) and intensive studying (Draganski et al., 2006). Implicit memory refers to intrinsic knowledge about how to perform an action and includes language learning (Mechelli et al., 2005), juggling (Draganski et al., 2004), mirror reading (Ilg et al., 2008), and musical training (Gaser and Schlaug, 2003). The $x, y$, and $z$ coordinates reported in these studies corresponding to increased density in brain regions were compiled and used to generate an activation likelihood estimate (ALE) map (www.brainmap.org). Coordinates are displayed on an average cortical surface in standard stereotactic space using SurfStat (http://www.math.mcgill.ca/keith/surfstat/). The ALE map reflects the probabilistic likelihood of increased gray matter density occurring within the six studies. Results demonstrate that although structural changes occur in functional areas related to the task, increases also occur in associative areas such as the posterior parietal and temporal cortices. Furthermore, studies examining explicit learning showed an overlap of increased gray matter density in the hippocampal gyrus.

sity, and the average intensity calculated for the gray matter partition is based on a mixture of different cell types, which cannot be differentiated with current techniques. Therefore, morphometric changes in density are somewhat difficult to interpret. Generally, the molecular correlates of gray matter density alterations are believed to be linked to changes in cell size, growth of neurons or glia, synaptogenesis, and even changes in blood flow or interstitial fluids (May, 2008). Central to the findings of Ilg et al. (2008) is that increased functional activity leads to intracortical remodeling. Whether those changes reflect molecular alterations in neurons or glia is hard to ascertain, because capillaries are also found in gray matter, and their density can vary with metabolic demands. In fact, animal studies have suggested that increased synaptic activity can elicit compensatory angiogenesis without necessarily producing new synapses (Black et al., 1990). Potential variations in capillary density are rarely discussed in relation to morphometric brain alterations, although they have been observed during functional activation in living human brains (Kuwabara et al., 1992). Consequently, the use of complementary methods, such as cortical evoked potentials recorded after training, might be a reasonable addition to rule out such causes when an increase in gray matter density is observed (May et al., 2007).

Ilg et al. (2008) showed increases in gray matter density induced after only short daily practice sessions. Additionally, changes corresponded to the location of practice-specific functional activation and not to the global extent of activation associated with the task. In light of these findings, the authors postulate that the gray matter density changes were linked to modifications of the axonal architecture and are unlikely attributable to the production of neurons or glia. Indeed, morphological changes in the brain have been observed over a period as short as $5 \mathrm{~d}$ of low-frequency transcranial magnetic stimulation (May et al., 2007), indicating that fast adjusting processes such as synaptic remodeling appear to underlie the 
anatomical changes. Neurogenesis as a potential underlying mechanism may not be entirely ruled out because many studies in vertebrates have demonstrated the production of new neurons throughout adulthood, particularly in the hippocampal formation, and show that these newly generated neurons are able to form connections with the CA3 region as soon as 1 or 2 weeks after mitosis (Gould et al., 1999).

In conjunction with these new morphometric techniques, complementary research is needed in animal models to understand the exact molecular events underlying experience-dependent plasticity seen in humans. Indeed, our interpretation of the various findings can hardly be conclusive until learning-dependent morphometric changes observed in animals are coupled with histological and immunological data. Nevertheless, this new exciting field of research undoubtedly highlights the remarkable potential of the adult brain to undergo anatomical changes that have a great impact on its functioning. Improved understanding of experience-dependent changes in cortical plasticity has vast clinical implications for neurorehabilitation programs after stroke, as well as for treatments of chronic pain that focus on use-dependent plasticity to improve mobility and alleviate pain.

\section{References}

Ashburner J, Friston KJ (2000) Voxel-based morphometry-the methods. Neuroimage 11:805-821.

Black JE, Isaacs KR, Anderson BJ, Alcantara AA, Greenough WT (1990) Learning causes synaptogenesis, whereas motor activity causes angiogenesis, in cerebellar cortex of adult rats. Proc Natl Acad Sci U S A 87:5568-5572.

Draganski B, Gaser C, Busch V, Schuierer G, Bogdahn U, May A (2004) Neuroplasticity: changes in grey matter induced by training. Nature 427:311-312.

Draganski B, Gaser C, Kempermann G, Kuhn HG, Winkler J, Büchel C, May A (2006) Temporal and spatial dynamics of brain structure changes during extensive learning. J Neurosci 26:6314-6317.

Gaser C, Schlaug G (2003) Brain structures differ between musicians and non-musicians. J Neurosci 23:9240-9245.

Gould E, Beylin A, Tanapat P, Reeves A, Shors TJ (1999) Learning enhances adult neurogenesis in the hippocampal formation. Nat Neurosci 2:260-265.

Ilg R, Wohlschläger AM, Gaser C, Liebau Y, Dauner R, Wöller A, Zimmer C, Zihl J, Mühlau M (2008) Gray matter increase induced by practice correlates with task-specific activation: a combined functional and morphometric magnetic resonance imaging study. J Neurosci 28:4210-4215.

Kuwabara H, Ohta S, Brust P, Meyer E, Gjedde A (1992) Density of perfused capillaries in living human brain during functional activation. Prog Brain Res 91:209-215.

Maguire EA, Gadian DG, Johnsrude IS, Good CD, Ashburner J, Frackowiak RS, Frith CD (2000) Navigation-related structural change in the hippocampi of taxi drivers. Proc Natl Acad Sci U S A 97:4398-4403.

May A (2008) Chronic pain may change the structure of the brain. Pain 137:7-15.

May A, Hajak G, Gänssbauer S, Steffens T, Langguth B, Kleinjung T, Eichhammer P (2007) Structural brain alterations following 5 days of intervention: dynamic aspects of neuroplasticity. Cereb Cortex 17:205-210.

Mechelli A, Friston KJ, Frackowiak RS, Price CJ (2005) Structural covariance in the human cortex. J Neurosci 25:8303-8310.

Merzenich MM, Sameshima K (1993) Cortical plasticity and memory. Curr Opin Neurobiol 3:187-196. 